\title{
GLOBAL EXISTENCE FOR A KINETIC MODEL OF CHEMOTAXIS VIA DISPERSION AND STRICHARTZ ESTIMATES.
}

\author{
NIKOLAOS BOURNAVEAS, VINCENT CALVEZ, SUSANA GUTIÉRREZ, \\ AND BENOÎT PERTHAME
}

\begin{abstract}
We investigate further the existence of solutions to kinetic models of chemotaxis. These are nonlinear transport-scattering equations with a quadratic nonlinearity which have been used to describe the motion of bacteria since the 80 's when experimental observations have shown they move by a series of 'run and tumble'. The existence of solutions has been obtained in several papers CMPS, HKS1, HKS3, using direct and strong dispersive effects.

Here, we use the weak dispersion estimates of $[\mathrm{CP}]$ to prove global existence in various situations depending on the turning kernel. In the most difficult cases, where both the velocities before and after tumbling appear, with the known methods, only Strichartz estimates can give a result, with a smallness assumption.
\end{abstract}

\section{INTRODUCTION}

In this paper we study the Othmer-Dunbar-Alt kinetic model of chemotaxis and prove global existence of solutions under various assumptions on the turning kernel. This model was proposed in [Alt, ODA] for the description of the chemotactic movement of cells in the presence of a chemical substance and it can be thought of as the mesoscopic analogue of the famous Keller-Segel model [KS1, KS2, KS3, H]. It was proposed in the 80 's, after the experimental observation that bacteria ( $E$. Coli in the present case, but this is also true for other bacteria as B. Subtilis for instance) move by a series of 'run and tumble' corresponding to the clockwise

Date: October 30, 2018.

1991 Mathematics Subject Classification. 92C17 (82B40 92B05) .

Key words and phrases. kinetic equations, chemotaxis, dispersion estimates, Strichartz estimates. 
or counterclockwise activations of their flagellas in response to chemoattractant substances and receptors saturation.

Denoting the cell density by $f(t, x, v)$ and the density of the chemoattractant by $S(t, x)$ the equations read as follows:

$$
\begin{aligned}
& \partial_{t} f+v \cdot \nabla_{x} f=\int_{V}\left(T[S] f^{\prime}-T^{*}[S] f\right) d v^{\prime}, \\
& f(0, x, v)=f_{0}(x, v) \\
& \beta S-\Delta S=\rho:=\int_{V} f(t, x, v) d v, \beta=0,1 .
\end{aligned}
$$

We have used the abbreviations $\int_{V} T[S] f^{\prime} d v^{\prime}=\int_{V} T[S]\left(t, x, v, v^{\prime}\right) f\left(t, x, v^{\prime}\right) d v^{\prime}$ and $\int_{V} T^{*}[S] f d v^{\prime}=\int_{V} T[S]\left(t, x, v^{\prime}, v\right) f(t, x, v) d v^{\prime}$. The velocity space $V$ is assumed to be a bounded three dimensional domain, typical examples being balls $\{|v| \leq R\}$ and spherical shells $\{r \leq|v| \leq R\}$. As a consequence, if $f_{0}$ has compact support in $x$, this will be so for all later times, and many aspects of the present paper can be simplified or improved, but of course to the expense of generality. Therefore we do not go in that direction.

Several earlier works have been devoted to the mathematical study of this kinetic model of chemotaxis. In [HO], the linear system has been studied (i.e. with a given field $S$ ) and in particular a major issue has been exhibited concerning the 'memory' effect present in the model through a time scale $\epsilon$ in expressions as $S\left(x-\epsilon v^{\prime}\right)$ or $S(x+\epsilon v)$. Not only this is a major experimental observation related to receptors saturation, but it also is responsible for an asymmetric kernel (in $v, v^{\prime}$ ) which yields the drift term in the Keller-Segel model that is derived in the diffusion limit of equation (1.1). The meaning of $S\left(t, x-\epsilon v^{\prime}\right)$ is that cells measure the concentration of the chemical $S$ at position $x-\epsilon v^{\prime}$ before changing their direction at position $x$, because of an internal memory effect. The other contribution $S(t, x+\epsilon v)$ is interpreted as follows: cells are able to measure the concentration at a location $x+\epsilon v$ thanks to sensorial protrusions. We set $\epsilon=1$ in the following without loss of generality.

The nonlinear Initial Value Problem (1.1) was first studied in CMPS where global existence was proved in $d=3$ dimensions under the assumption that the 
turning kernel satisfies the condition

$$
0 \leq T[S]\left(t, x, v, v^{\prime}\right) \leq C\left(1+S(t, x+v)+S\left(t, x-v^{\prime}\right)\right)
$$

and the initial data satisfy $0 \leq f_{0} \in L^{1}\left(\mathbb{R}^{6}\right) \cap L^{\infty}\left(\mathbb{R}^{6}\right)$. The proof starts with the fact that the $L_{x, v}^{1}$-norm of the solution $f$ is a-priori bounded thanks to conservation of mass

$$
\iint_{V} f(t, x, v) d v d x=\iint_{V} f_{0}(x, v) d v d x=: \mathbf{M},
$$

and then proceeds to bootstrap higher $L_{x, v}^{p}$-norms based on strong dispersion estimates (see [GL, P1]).

The same method was used in the paper HKS1 which points out the difference in the dispersive arguments for terms involving both $S(t, x+v)$ and $S\left(t, x-v^{\prime}\right)$. The authors prove global existence in $d=3$ dimensions under the assumption

$$
0 \leq T[S]\left(t, x, v, v^{\prime}\right) \leq C(1+S(t, x+v)+|\nabla S(t, x+v)|)
$$

or

$$
0 \leq T[S]\left(t, x, v, v^{\prime}\right) \leq C\left(1+S\left(t, x-v^{\prime}\right)+\left|\nabla S\left(t, x-v^{\prime}\right)\right|\right)
$$

and in $d=2$ dimensions together with $\beta=1$ under the assumption

$$
\begin{aligned}
0 \leq T[S]\left(t, x, v, v^{\prime}\right) \leq C(1+S(t, x+v)+ & S\left(t, x-v^{\prime}\right) \\
& \left.+|\nabla S(t, x+v)|+\left|\nabla S\left(t, x-v^{\prime}\right)\right|\right) .
\end{aligned}
$$

The main difficulty appears: scattering terms involving $S\left(x-v^{\prime}\right)$ or $S(x+v)$ lead to use two different dispersion estimates, that lead to use a bootstrap with integrability exponents that are only compatible in dimensions less than four. The same dispersive method has been pushed forward in [HKS1, HKS3], including more general biologically relevant turning kernels and pointing out several limitations.

For more results and models involving kinetic equations, see [FLP, CR, HPS], for hyperbolic models [HKS2, [EH] and for surveys on the kinetic aspects [P1, [P2].

In this paper we use the dispersion and Strichartz estimates for solutions of the kinetic transport equation proved in $[\mathrm{CP}$ to extend the three dimensional results of [CMPS] and [HKS1] to more general turning kernels. Compared to [CMPS] and HKS1] where $L_{x, v}^{p}$-spaces are used, the main feature of our present estimates is to work in $L_{x}^{p} L_{v}^{q}$-spaces for appropriate choices of $p$ and $q$ (see remark 3.3 for instance). 
In our first result we combine the dispersion estimate of [CP] with the wellknown consequence of Calderón-Zygmund theory that any second derivative can be controlled in $L^{p}(1<p<\infty)$ by the Laplacian in $L^{p}$, to prove global existence for the IVP (1.1) under assumption (1.3) below. The latter assumption allows the turning kernel $T[S]$ to be controlled by second derivatives of the chemoattractant density $S$. Notice that this result is valid in all dimensions $d \geq 2$.

Theorem 1. Let $d \geq 2$ and suppose that the (continuous) turning kernel satisfies

$$
0 \leq T[S]\left(t, x, v, v^{\prime}\right) \leq C\left(1+\sum_{|\alpha| \leq 2}\left|\partial^{\alpha} S(t, x+v)\right|\right)
$$

Fix $p \in\left(1, \frac{d}{d-1}\right)$. If the initial data $f_{0} \in L^{1}\left(\mathbb{R}^{2 d}\right)$ is nonnegative and such that $\left\|f_{0}(x-t v, v)\right\|_{L^{p}\left(\mathbb{R}_{x}^{d} ; L^{1}\left(\mathbb{R}_{v}^{d}\right)\right)}$ is finite for all $t>0$ (1), then the Cauchy problem (1.1) with $\beta=1$ has a global weak solution $f$ with $f(t) \in L^{1}\left(\mathbb{R}^{2 d}\right) \cap L^{p}\left(\mathbb{R}_{x}^{d} ; L^{1}(V)\right)$ for all $t \geq 0$.

As it was already commented in [CMPS] and [HKS1] it is difficult to mix terms involving $x+v$ with terms involving $x-v^{\prime}$. In this direction we shall prove the following result.

Theorem 2. Let $d=3$ and suppose that the (continuous) turning kernel satisfies

$$
0 \leq T[S]\left(t, x, v, v^{\prime}\right) \leq C\left(1+S(t, x+v)+S\left(t, x-v^{\prime}\right)+|\nabla S(t, x+v)|\right) .
$$

Let $q \in(1,3 / 2)$. Then there exists a $p \in(3 / 2,3)$ (depending on $q)$ such that if the initial data $f_{0} \in L^{1}\left(\mathbb{R}^{6}\right)$ is nonnegative and such that $\left\|f_{0}(x-t v, v)\right\|_{L^{p}\left(\mathbb{R}_{x}^{3} ; L^{q}\left(\mathbb{R}_{v}^{3}\right)\right)}$ is finite for all $t>0$, then the Cauchy problem (1.1) has a global weak solution $f$ with $f(t) \in L^{1}\left(\mathbb{R}^{6}\right) \cap L^{p}\left(\mathbb{R}_{x}^{3} ; L^{q}(V)\right)$ for all $t \geq 0$.

Hypothesis (1.4) does not allow putting together the two gradients $\nabla S(t, x+v)$ and $\nabla S\left(t, x-v^{\prime}\right)$. However, our next result shows that if we add the assumption that the critical $L_{x, v}^{3 / 2}$-norm of the initial data is sufficiently small then we have global existence under a very general hypothesis on the turning kernel, see (1.5)

\footnotetext{
${ }^{1}$ This assumption means simply that the solution $f(t, x, v)=f_{0}(x-t v, v)$ of the linear homogeneous kinetic transport equation with initial data $f_{0}$ belongs to $L_{x}^{p} L_{v}^{1}$ for all times. This assumption is satisfied if for example $f_{0}$ is $L_{x}^{1} L_{v}^{p}$ (see proposition 2.1).
} 
and the even weaker (4.1). The proof uses the Strichartz estimates of [CP] and can be made to work in $d=2$ and 4 dimensions too, see Remark 4.1

Theorem 3. Let $d=3$. Consider nonnegative initial data $f_{0} \in L^{1}\left(\mathbb{R}^{2 d}\right) \cap L^{a}\left(\mathbb{R}^{2 d}\right)$, where $\frac{3}{2} \leq a \leq 2$, and assume that $\left\|f_{0}\right\|_{L^{a}\left(\mathbb{R}^{2 d}\right)}$ is sufficiently small. Assume that the (continuous) turning kernel $T[S]$ satisfies the condition

$0 \leq T[S]\left(t, x, v, v^{\prime}\right) \lesssim|S(t, x \pm v)|+\left|S\left(t, x \pm v^{\prime}\right)\right|+|\nabla S(t, x \pm v)|+\left|\nabla S\left(t, x \pm v^{\prime}\right)\right|$

where any combination of signs is allowed in the right hand side. Then the IVP (1.1) with $\beta=1$ has a global weak solution $f \in L_{t}^{3}\left([0, \infty) ; L^{p}\left(\mathbb{R}_{x}^{3} ; L^{q}(V)\right)\right)$, where $\frac{1}{p}=\frac{1}{a}-\frac{1}{9}$ and $\frac{1}{q}=\frac{1}{a}+\frac{1}{9}$. This result also holds if hypothesis (1.5) is replaced by the weaker (4.1) below.

\section{Dispersion and Strichartz estimates}

In this section we collect the dispersion and Strichartz estimates we shall use later. We start with the dispersion estimate.

Proposition 2.1. (Dispersion estimate, $[\mathrm{CP})$ Let $f_{0} \in L^{q}\left(\mathbb{R}_{x}^{d} ; L^{p}\left(\mathbb{R}_{v}^{d}\right)\right)$ where $1 \leq$ $q \leq p \leq \infty$, and let $f$ solve

$$
\partial_{t} f+v \cdot \nabla_{x} f=0
$$

with initial data $f(0, x, v)=f_{0}(x, v)$. Then

$$
\|f(t)\|_{L^{p}\left(\mathbb{R}_{x}^{d} ; L^{q}\left(\mathbb{R}_{v}^{d}\right)\right)} \leq \frac{1}{|t|^{\left(\frac{1}{q}-\frac{1}{p}\right)}}\left\|f_{0}\right\|_{L^{q}\left(\mathbb{R}_{x}^{d} ; L^{p}\left(\mathbb{R}_{v}^{d}\right)\right)} .
$$

We are going to need the following two versions of the dispersion estimate. First of all observe that the solution of (2.1) with initial data $f_{0}(x, v)$ is simply $f(t, x, v)=$ $f_{0}(x-t v, v)$. Therefore the dispersion estimate says that for any function $h \in$ $L^{q}\left(\mathbb{R}_{x}^{d} ; L^{p}\left(\mathbb{R}_{v}^{d}\right)\right)$, where $1 \leq q \leq p \leq \infty$, we have

$$
\|h(x-t v, v)\|_{L^{p}\left(\mathbb{R}_{x}^{d} ; L^{q}\left(\mathbb{R}_{v}^{d}\right)\right)} \leq \frac{1}{|t|^{\left(\frac{1}{q}-\frac{1}{p}\right)}}\|h(x, v)\|_{L^{q}\left(\mathbb{R}_{x}^{d} ; L^{p}\left(\mathbb{R}_{v}^{d}\right)\right)} .
$$

Replacing $h(x, v)$ by $h(x, v) \mathbb{1}_{V}(v)$ we get

$$
\|h(x-t v, v)\|_{L^{p}\left(\mathbb{R}_{x}^{d} ; L^{q}(V)\right)} \leq \frac{1}{|t|^{d\left(\frac{1}{q}-\frac{1}{p}\right)}}\|h(x, v)\|_{L^{q}\left(\mathbb{R}_{x}^{d} ; L^{p}(V)\right)} .
$$


In the special case of a function $h(x)$ which is independent of $v$ we get

$$
\|h(x-t v)\|_{L^{p}\left(\mathbb{R}_{x}^{d} ; L^{q}(V)\right)} \leq \frac{C(|V|)}{|t|^{d\left(\frac{1}{q}-\frac{1}{p}\right)}}\|h(x)\|_{L^{q}\left(\mathbb{R}_{x}^{d}\right)} .
$$

Next we recall the Strichartz estimates of $[\mathrm{CP}$.

Proposition 2.2. (Strichartz estimates, $[\mathrm{CP})$ Let $d \geq 2$ and let $r, p, q, a \in[1, \infty]$ satisfy the conditions

$$
p \geq q, \quad \frac{2}{r}=d\left(\frac{1}{q}-\frac{1}{p}\right)<1, \quad a=H M(p, q) \leq 2,
$$

where $H M$ denotes the harmonic mean.

(1) If $f(t, x, v)$ solves

$$
\partial_{t} f+v \cdot \nabla_{x} f=g \quad, \quad f(0, x, v)=0
$$

then

$$
\|f\|_{L_{t}^{r} L_{x}^{p} L_{v}^{q}} \leq C\|g\|_{L_{t}^{r^{\prime}} L_{x}^{q} L_{v}^{p}}
$$

(2) If $f(t, x, v)$ solves

$$
\partial_{t} f+v \cdot \nabla_{x} f=0 \quad, \quad f(0, x, v)=f_{0}(x, v),
$$

then

$$
\|f\|_{L_{t}^{r} L_{x}^{p} L_{v}^{q}} \leq C\left\|f_{0}\right\|_{L_{x, v}^{a}}
$$

\section{Global existence for arbitrarily large Data}

In this Section we prove Theorems 1 and 2, We start with Theorem 1, Using the dispersion estimate gives rise to two norms, $\left\|\partial^{\alpha} S\right\|_{L^{p}}$ and $\|\rho\|_{L^{q}}$, see (3.2). Of course each of them could be estimated in terms of $f$, but this would result in a quadratic term and would make the use of Gronwall's inequality impossible. However, thanks to conservation of mass, we can choose $q=1$ (this corresponds to velocity averaging) and bound $\|\rho\|_{L^{q}}$ a-priori. The norm $\left\|\partial^{\alpha} S\right\|_{L^{p}}$ is then estimated using the well-known Calderón-Zygmund inequality if $|\alpha|=2$, see (3.4) (see also Remark 3.4 at the end of this Section) and Young inequality if $|\alpha| \leq 1$.

We use the standard abbreviations for mixed spaces, for example $L_{x}^{p} L_{v}^{q}$ stands for $L^{p}\left(\mathbb{R}_{x}^{d} ; L_{v}^{q}(V)\right)$. In all cases $x$ varies in the whole space $\mathbb{R}^{d}$ while $v$ and $v^{\prime}$ are restricted in the bounded velocity space $V$. 
Proof of Theorem 1. Fix $p$ and $q$ with $1 \leq q \leq p \leq \infty$. Arguing as in CMPS] we have

$$
\begin{aligned}
f(t, x, v) & \leq f_{0}(x-t v, v)+C \int_{0}^{t} \rho(t-s, x-s v) d v \\
& +C \sum_{|\alpha| \leq 2} \int_{0}^{t}\left|\partial^{\alpha} S(t-s, x-s v+v)\right| \rho(t-s, x-s v) d s
\end{aligned}
$$

therefore, using the dispersion estimate (2.3), we have

$$
\begin{aligned}
& \|f(t, x, v)\|_{L_{x}^{p} L_{v}^{q}} \leq\left\|f_{0}(x-t v, v)\right\|_{L_{x}^{p} L_{v}^{q}}+C(|V|) \int_{0}^{t} \frac{1}{s^{d\left(\frac{1}{q}-\frac{1}{p}\right)}}\|\rho(t-s, \cdot)\|_{L^{q}} d s \\
& +C \sum_{|\alpha| \leq 2} \int_{0}^{t} \frac{1}{s^{d\left(\frac{1}{q}-\frac{1}{p}\right)}}\left\|\partial^{\alpha} S(t-s, x+v) \rho(t-s, x)\right\|_{L_{x}^{q} L_{v}^{p}} d s \\
& \leq C_{0}(t)+C(|V|) \int_{0}^{t} \frac{1}{s^{d\left(\frac{1}{q}-\frac{1}{p}\right)}}\|\rho(t-s, \cdot)\|_{L^{q}} d s \\
& +C \sum_{|\alpha| \leq 2} \int_{0}^{t} \frac{1}{s^{d\left(\frac{1}{q}-\frac{1}{p}\right)}}\left\|\partial^{\alpha} S(t-s, \cdot)\right\|_{L^{p}}\|\rho(t-s, \cdot)\|_{L^{q}} d s
\end{aligned}
$$

where we have set $C_{0}(t)=\left\|f_{0}(x-t v, v)\right\|_{L_{x}^{p} L_{v}^{q}}$. Choose $q=1$ and $p \in\left(1, \frac{d}{d-1}\right)$. Then by conservation of mass $\|\rho(t-s, \cdot)\|_{L^{q}}=M$. Using Young's inequality and conservation of mass for the derivatives of order one, we have

$$
\|\nabla S(t-s, \cdot)\|_{L^{p}}=C\|\rho(t-s, \cdot) * \nabla G\|_{L^{p}} \leq\|\rho(t-s, \cdot)\|_{L^{1}}\|\nabla G\|_{L^{p}}=C M
$$

where $G(x)=\frac{1}{4 \pi} \int_{0}^{\infty} e^{-\pi \frac{|x|^{2}}{4 s}-\frac{s}{4 \pi}} s^{\frac{-d+2}{2}} \frac{d s}{s}$ is the Bessel potential, and we get a similar estimate for $S$. For the derivatives of order two we have ([] $]$, p. 59, Proposition 3)

$$
\begin{aligned}
\left\|\partial_{i j} S(t-s)\right\|_{L^{p}} \leq C(d, p)\|\Delta S(t-s)\|_{L^{p}} \leq & C(d, p)\|\rho(t-s)\|_{L^{p}}+C\|S(t-s)\|_{L^{p}} \\
& \leq C(d, p)\|\rho(t-s)\|_{L^{p}}+C M
\end{aligned}
$$

Therefore (3.2) gives

$$
\|\rho(t)\|_{L^{p}} \leq C_{1}(t)+C(d, p, M) \int_{0}^{t} \frac{1}{s^{d / p^{\prime}}}\|\rho(t-s)\|_{L^{p}} d s .
$$

Since $d / p^{\prime}<1$, we can use Gronwall's inequality to get

$$
\|\rho(t)\|_{L^{p}} \leq C\left(d, p, t, f_{0}\right) .
$$

This completes the a-priori estimates. See remark 4.2 . 
Remark 3.1. We have chosen $\beta=1$ so that our $S$ decays sufficiently fast in order to apply the Calderón-Zygmund inequality. If $\beta=0$ we have $S=S^{s}+S^{l} \in L^{p}+L^{\infty}$ and we have no decay for $S^{l}$.

The proof of Theorem 2 uses the dispersion estimate of Proposition 2.1 as well as Young's convolution inequality and the Hardy-Littlewood-Sobolev inequality. The dispersion estimate is used to handle functions of $x-s v$ and $v$ which arise when we integrate the kinetic equation (1.1a), see (3.6) and (3.7) below. Each term in the right hand side of hypothesis (1.4) requires an estimate in $L_{x}^{p} L_{v}^{q}$ for a certain range of $p$ and $q$. Terms involving $x+v$ usually require small $p$ while terms involving $x-v^{\prime}$ require large $p$. The main difficulty then is to find one set of parameters that makes both estimates work. To deal with this we will view the term $\nabla S(t-s, x-s v+v) \rho(t-s, x-s v)$ as $\nabla S(t-s, x-(s-1) v) \rho(t-s, x-(s-1) v-v)$. This shifting of the singularity from $s=0$ to $s=1$ (see (3.9) ) results in a redistribution of norms that allows us to estimate the terms involving $\nabla S(x+v)$ and $S(x+v)$ without any restrictions on the parameter $p$, and it creates enough freedom so that, when we come to the more complicated estimates for $S\left(x-v^{\prime}\right)$, we are able to find a pair $(p, q)$ that works for both.

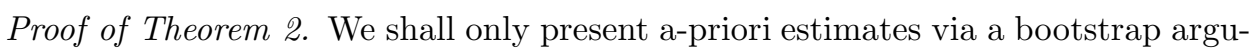
ment for the solution $f$ of (1.1) in the space $L^{p}\left(\mathbb{R}_{x}^{3} ; L_{v}^{q}(V)\right)$. The existence part of Theorem 2 then follows by well-known methods, see Remark 4.2. We present the proof in the more difficult case $\beta=0$.

Observe that $S=S^{s}+S^{l}$ where $S^{s}(t)=\frac{1}{4 \pi} \rho(t) * \frac{\mathbb{1}_{|x|<1}}{|x|}$ and $S^{l}(t)=\frac{1}{4 \pi} \rho(t) * \frac{\mathbb{1}_{|x|>1}}{|x|}$. The long part $S^{l}(t)$ is a-priori bounded thanks to conservation of mass:

$$
\left|S^{l}(t, x)\right| \leq C\|\rho(t)\|_{L^{1}}\left\|\frac{\mathbb{1}_{|x| \geq 1}}{|x|}\right\|_{L^{\infty}} \leq C M .
$$

Similarly we split $\nabla S$ as $\nabla S=(\nabla S)^{s}+(\nabla S)^{l}$ where $(\nabla S)^{s}(t)=\frac{1}{4 \pi} \rho(t) * \frac{\mathbb{1}_{|x| \leq 1}}{|x|^{2}}$ and $(\nabla S)^{l}(t)=\frac{1}{4 \pi} \rho(t) * \frac{\mathbb{1}_{|x| \geq 1}}{|x|^{2}}$ and show that $(\nabla S)^{l}$ is a-priori bounded. It follows that we may replace hypothesis (1.4) by

$$
0 \leq T[S]\left(t, x, v, v^{\prime}\right) \leq C\left(1+S^{s}(t, x+v)+S\left(t, x-v^{\prime}\right)+\left|(\nabla S)^{s}(t, x+v)\right|\right)
$$

where the new constant $C$ depends on the mass $M$. For technical reasons it is more convenient not to split $S\left(t, x-v^{\prime}\right)$. Following the reasoning in [CMPS] we estimate 
$f$ as follows:

$$
f(t, x, v) \leq C f_{0}(x-t v, v)+C \int_{0}^{t} \rho(t-s, x-s v) d s+C \sum_{j=1}^{3} f_{j}(t, x, v)
$$

where

$$
\begin{aligned}
f_{1}(t, x, v) & =\int_{0}^{t} \int_{V} S^{s}(t-s, x-s v+v) f\left(t-s, x-s v, v^{\prime}\right) d v^{\prime} d s \\
& =\int_{0}^{t} S^{s}(t-s, x-s v+v) \rho(t-s, x-s v) d s \\
f_{2}(t, x, v) & =\int_{0}^{t} \int_{V} S\left(t-s, x-s v-v^{\prime}\right) f\left(t-s, x-s v, v^{\prime}\right) d v^{\prime} d s \\
f_{3}(t, x, v) & =\int_{0}^{t}\left|(\nabla S)^{s}(t-s, x-s v+v)\right| \rho(t-s, x-s v) d s
\end{aligned}
$$

Fix $p$ and $q$ with $q \in[1,3 / 2)$ such that

$$
\lambda:=3\left(\frac{1}{q}-\frac{1}{p}\right)<1,1 \leq q \leq p \leq \infty
$$

These restrictions on $p$ and $q$ will be enough for all estimates involving $f_{1}$ and $f_{3}$ but more restrictions will be imposed later when we estimate $f_{2}$ and we will want to know that there is a pair $(p, q)$ that satisfies all of them (2). We start our estimates with $f_{3}$. We have

$$
\begin{aligned}
& \left\|f_{3}(t, x, v)\right\|_{L_{x}^{p} L_{v}^{q}} \leq \int_{0}^{t}\left\|(\nabla S)^{s}(t-s, x-s v+v) \rho(t-s, x-s v)\right\|_{L_{x}^{p} L_{v}^{q}} d s \\
& =\int_{0}^{t}\left\|(\nabla S)^{s}(t-s, x-(s-1) v) \rho(t-s, x-(s-1) v-v)\right\|_{L_{x}^{p} L_{v}^{q}} d s .
\end{aligned}
$$

For fixed $t \geq 0$ and $s \in(0, t)$ we use the dispersion estimate (2.4) with $t$ replaced by $s-1$ and $h(x, v)=\left|(\nabla S)^{s}(t-s, x)\right| \rho(t-s, x-v)$ to get

$$
\begin{aligned}
\left\|f_{3}(t, x, v)\right\|_{L_{x}^{p} L_{v}^{q}} & \leq \int_{0}^{t} \frac{1}{|s-1|^{\lambda}}\left\|(\nabla S)^{s}(t-s, x) \rho(t-s, x-v)\right\|_{L_{x}^{q} L_{v}^{p}} d s \\
& \leq \int_{0}^{t} \frac{1}{|s-1|^{\lambda}}\left\|(\nabla S)^{s}(t-s, \cdot)\right\|_{L^{q}}\|\rho(t-s, \cdot)\|_{L^{p}} d s .
\end{aligned}
$$

Because $q<3 / 2$ the quantity $\left\|(\nabla S)^{s}(t-s, \cdot)\right\|_{L^{q}}$ is uniformly bounded. Indeed, using Young's inequality we have

$$
\left\|(\nabla S)^{s}(t-s, \cdot)\right\|_{L^{q}} \leq C\|\rho(t-s, \cdot)\|_{L^{1}}\left\|\frac{\mathbb{1}_{|x| \leq 1}}{|x|^{2}}\right\|_{L^{q\left(\mathbb{R}^{3}\right)}} \leq C(q) M .
$$

\footnotetext{
${ }^{2}$ One choice of parameters that works is: $p=\frac{9}{5}, q=\frac{9}{7}$.
} 
On the other hand, since the velocity space is bounded, we have

$$
\|\rho(t-s, \cdot)\|_{L^{p}}=\|f(t-s, x, v)\|_{L_{x}^{p} L_{v}^{1}} \leq C(|V|, q)\|f(t-s, x, v)\|_{L_{x}^{p} L_{v}^{q}} .
$$

We conclude that

$$
\left\|f_{3}(t, x, v)\right\|_{L_{x}^{p} L_{v}^{q}} \leq C(|V|, q) M \int_{0}^{t} \frac{1}{|s-1|^{\lambda}}\|f(t-s, x, v)\|_{L_{x}^{p} L_{v}^{q}} d s .
$$

The estimate for $f_{1}$ is almost exactly the same. The only difference is that in the a-priori estimate (3.11) the norm $\left\|\frac{\mathbb{1}_{|x| \leq 1}}{|x|^{2}}\right\|_{L^{q}\left(\mathbb{R}^{3}\right)}$ is replaced by $\left\|\frac{\mathbb{1}_{|x| \leq 1}}{|x|}\right\|_{L^{q}\left(\mathbb{R}^{3}\right)}$ which is again finite because $q<3 / 2<3$. We get:

$$
\left\|f_{1}(t, x, v)\right\|_{L_{x}^{p} L_{v}^{q}} \leq C(|V|, q) M \int_{0}^{t} \frac{1}{|s-1|^{\lambda}}\|f(t-s, x, v)\|_{L_{x}^{p} L_{v}^{q}} d s .
$$

Remark 3.2. Splitting in addition between small and long times $(s \gtrless 1 / 2$ for example) we end up with a priori estimates without any restriction on the exponent $p$. But this technical improvement is not relevant in this proof.

Next we estimate $f_{2}$. We start with some numerology which we explain later. Fix $q \in(1,3 / 2)$. There exists a $p \in(3 / 2,3)$ such that

$$
3\left(\frac{1}{q}-\frac{1}{p}\right)=1-\frac{3 p^{\prime}}{\left(q^{\prime}\right)^{2}} \text {. }
$$

To see this write (3.14) as $\delta(p):=1-\frac{3 p^{\prime}}{\left(q^{\prime}\right)^{2}}-3\left(\frac{1}{q}-\frac{1}{p}\right)=0$ and think of this expression as a continuous function of the variable $p \in[3 / 2,3]$. For $p=3 / 2$ we have $\delta(3 / 2)=1-\frac{9}{\left(q^{\prime}\right)^{2}}-3\left(\frac{1}{q}-\frac{2}{3}\right)=\frac{3\left(q^{\prime}-3\right)}{\left(q^{\prime}\right)^{2}}>0$. On the other hand for $p=3$ we have $\delta(3)=1-\frac{9}{2\left(q^{\prime}\right)^{2}}-3\left(\frac{1}{q}-\frac{1}{3}\right)<-\frac{9}{2\left(q^{\prime}\right)^{2}}<0$. The existence of $p$ follows. Notice that with this choice of $p$ and $q$ we still have $1 \leq q \leq p \leq \infty$ and moreover the integrability condition $\lambda<1$ is satisfied thanks to (3.14).

Remark 3.3. In fact we are allowed to choose $q$ and $p$ to be in the following set of exponents

$$
\mathcal{A}=\left\{p^{\prime} \geq 1, q^{\prime} \geq 1 \mid q^{\prime}>p^{\prime}, 3\left(\frac{1}{q}-\frac{1}{p}\right)+\frac{3 p^{\prime}}{\left(q^{\prime}\right)^{2}} \leq 1, \frac{1}{q}-\frac{1}{p}<\frac{1}{3}\right\} .
$$

This set of admissible exponents for the estimate of $\left\|f_{2}(t, x, v)\right\|_{L_{x}^{p} L_{v}^{q}}$ is plotted in figure 1 in the coordinates $\left(q^{\prime}, p^{\prime}\right)$. The key point is that it intersects the constrain $\left\{q^{\prime}>3\right\}$ which comes from the estimates on $f_{1}$ and $f_{3}$. This can be done only by decoupling $p$ and $q$.

Assuming some linear contribution of $\nabla S\left(x-v^{\prime}\right)$ in the turning kernel bound (1.4) 


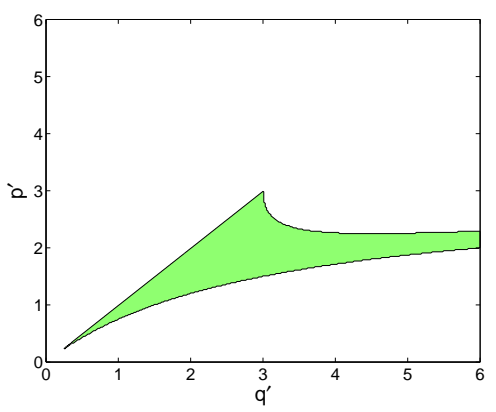

(a)

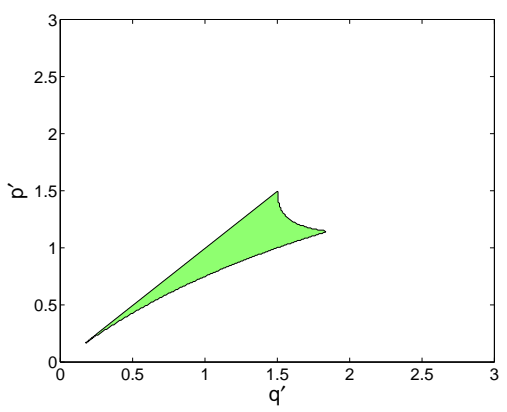

(b)

Figure 1. Set of admissible exponents $\left(q^{\prime}, p^{\prime}\right)$ for the estimate of $\left\|f_{2}(t, x, v)\right\|_{L_{x}^{p} L_{v}^{q}}$, corresponding to $S\left(x-v^{\prime}\right)$ (a) and $\nabla S\left(x-v^{\prime}\right)$

(b) respectively.

would have lead to the set represented in figure 1 b. The latter does not intersect the half-plane $\left\{q^{\prime}>3\right\}$.

Finally we define $\theta \in(0,1), c \in(1, q)$ and $b \in\left(1, c^{\prime}\right)$ by the following relations:

$$
\frac{1}{q}=1-\theta+\frac{\theta}{p}, \frac{1}{c}=1-\theta+\frac{\theta}{q}, \frac{1}{b}+\frac{1}{c}=\frac{5}{3} .
$$

Proceeding with the estimates we have

$$
\left\|f_{2}(t, x, v)\right\|_{L_{x}^{p} L_{v}^{q}} \leq \int_{0}^{t}\left\|\int_{V} S\left(t-s, x-s v-v^{\prime}\right) f\left(t-s, x-s v, v^{\prime}\right) d v^{\prime}\right\|_{L_{x}^{p} L_{v}^{q}} d s .
$$

For fixed $t \geq 0$ and $s \in(0, t)$ use the dispersion estimate (2.5) with

$$
h(x)=\int_{V} S\left(t-s, x-v^{\prime}\right) f\left(t-s, x, v^{\prime}\right) d v^{\prime}
$$

to get

$$
\begin{aligned}
\| \int_{V} S\left(t-s, x-s v-v^{\prime}\right) & f\left(t-s, x-s v, v^{\prime}\right) d v^{\prime} \|_{L_{x}^{p} L_{v}^{q}} \\
\leq & \frac{1}{s^{\lambda}}\left\|\int_{V} S\left(t-s, x-v^{\prime}\right) f\left(t-s, x, v^{\prime}\right) d v^{\prime}\right\|_{L_{x}^{q}} .
\end{aligned}
$$

By Hölder's inequality

$$
\int_{V} S\left(t-s, x-v^{\prime}\right) f\left(t-s, x, v^{\prime}\right) d v^{\prime} \leq\|S(t-s, \cdot)\|_{L_{x}^{c^{\prime}}}\left\|f\left(t-s, x, v^{\prime}\right)\right\|_{L_{v^{\prime}}^{\prime}} .
$$


Since $1<b<c^{\prime}<\infty$ and $\frac{1}{b}-\frac{1}{c^{\prime}}=\frac{2}{3}$ we can apply the Hardy-Littlewood-Sobolev inequality (see for instance [S], Theorem 1, page 199) to get

$$
\|S(t-s, \cdot)\|_{L^{c^{\prime}}}=C\left\|\rho(t-s, \cdot) * \frac{1}{|x|}\right\|_{L^{c^{\prime}}\left(\mathbb{R}^{3}\right)} \leq C\|\rho(t-s, \cdot)\|_{L^{b}} .
$$

It is easy to check that $1<b<p(3)$, therefore if we define $\epsilon \in(0,1)$ by

$$
\frac{1}{b}=1-\epsilon+\frac{\epsilon}{p}
$$

we can use interpolation and conservation of mass to obtain

$$
\|\rho(t-s)\|_{L^{b}} \leq\|\rho(t-s)\|_{L^{1}}^{1-\epsilon}\|\rho(t-s)\|_{L^{p}}^{\epsilon} \leq M^{1-\epsilon}\|\rho(t-s)\|_{L^{p}}^{\epsilon} .
$$

We have shown that

$$
\int_{V} S\left(t-s, x-v^{\prime}\right) f\left(t-s, x, v^{\prime}\right) d v^{\prime} \leq C M^{1-\epsilon}\|\rho(t-s, \cdot)\|_{L^{p}}^{\epsilon}\left\|f\left(t-s, x, v^{\prime}\right)\right\|_{L_{v^{\prime}}^{c}},
$$

and as a product we obtain

$$
\begin{aligned}
& \| \int_{V} S\left(t-s, x-v^{\prime}\right) f\left(t-s, x, v^{\prime}\right) d v^{\prime} \\
& \leq C M^{1-\epsilon}\|\rho(t-s, \cdot)\|_{L^{p}}^{\epsilon}\|f(t-s, x, v)\|_{L_{x}^{q} L_{v}^{c}} .
\end{aligned}
$$

We aim to interpolate the $L_{x}^{q} L_{v}^{c}$-norm between $L^{1} L^{1}$ and $L^{p} L^{q}$ in order to conclude with a Gronwall lemma. This is achieved thanks to the first two relations in (3.16).

$$
\begin{aligned}
\|f(t-s, x, v)\|_{L_{x}^{q} L_{v}^{c}} & \leq\|f(t-s, x, v)\|_{L_{x}^{1} L_{v}^{1}}^{1-\theta}\|f(t-s, x, v)\|_{L_{x}^{p} L_{v}^{q}}^{\theta} \\
& \leq M^{1-\theta}\|f(t-s, x, v)\|_{L_{x}^{p} L_{v}^{q}}^{\theta}
\end{aligned}
$$

Using this estimate together with $\|\rho(t-s, \cdot)\|_{L^{p}} \leq C(|V|, q)\|f(t-s, x, v)\|_{L_{x}^{p} L_{v}^{q}}$ into (3.22), we get

$$
\left\|\int_{V} S\left(t-s, x-v^{\prime}\right) f\left(t-s, x, v^{\prime}\right) d v^{\prime}\right\|_{L_{x}^{q}} \leq C(|V|, q) M^{2-(\epsilon+\theta)}\|f(t-s, x, v)\|_{L_{x}^{p} L_{v}^{q}}^{\epsilon+\theta} .
$$

$$
3 \frac{1}{b}-\frac{1}{p}=\frac{5}{3}-\frac{1}{c}-\frac{1}{p}=\frac{5}{3}-1+\theta-\frac{\theta}{q}-\frac{1}{p}=\left(\frac{2}{3}-\frac{1}{p}\right)+\frac{\theta}{q^{\prime}}>0 .
$$


We can now argue that we opted for (3.14) to ensure that $\epsilon+\theta=1$ (4). Therefore

$$
\left\|\int_{V} S\left(t-s, x-v^{\prime}\right) f\left(t-s, x, v^{\prime}\right) d v^{\prime}\right\|_{L_{x}^{q}} \leq C(|V|, q) M\|f(t-s, x, v)\|_{L_{x}^{p} L_{v}^{q}} .
$$

From (3.17), (3.18) and (3.25), we conclude that

$$
\left\|f_{2}(t, x, v)\right\|_{L_{x}^{p} L_{v}^{q}} \leq C(|V|, q) M \int_{0}^{t} \frac{1}{s^{\lambda}}\|f(t-s, x, v)\|_{L_{x}^{p} L_{v}^{q}} d s .
$$

This completes the estimate of $f_{2}$. Finally we have to estimate the first two terms in the right hand side of (3.6). For the first term we have by our hypothesis on the initial data that $\left\|f_{0}(x-t v, v)\right\|_{L_{x}^{p} L_{v}^{q}}=: C_{0}(t)<\infty$ for all $t$. We can use dispersion and interpolation for the second term, leading to

$$
\left\|\int_{0}^{t} \rho(t-s, x-s v) d s\right\|_{L_{x}^{p} L_{v}^{q}} \leq \int_{0}^{t} s^{-\lambda}\|\rho(t-s)\|_{L_{x}^{q}} d s \leq C \int_{0}^{t} s^{-\lambda}\|\rho(t-s)\|_{L_{x}^{p}}^{\theta} d s,
$$

where $\theta$ has already been defined in (3.16).

Putting everything together we conclude that

$$
\begin{array}{rl}
\|f(t, x, v)\|_{L_{x}^{p} L_{v}^{q}} \leq C_{0}(t)+C(|V|, q) M \int_{0}^{t} & K(s)\|f(t-s, x, v)\|_{L_{x}^{p} L_{v}^{q}} d s \\
& +C \int_{0}^{t} s^{-\lambda}\|f(t-s)\|_{L_{x}^{p} L_{v}^{q}}^{\theta} d s,
\end{array}
$$

where $K(s)=1+\frac{1}{s^{\lambda}}+\frac{1}{|s-1|^{\lambda}}$. Since $\lambda<1$ we can apply Gronwall's inequality to obtain

$$
\|f(t, x, v)\|_{L_{x}^{p} L_{v}^{q}} \leq C\left(|V|, q, t, f_{0}\right) .
$$

Remark 3.4. It would be interesting to know whether, in the case $\beta=1$, the hypotheses (1.4) and (1.3) can be combined into the single assumption:

$$
\begin{aligned}
0 \leq T[S]\left(t, x, v, v^{\prime}\right) \leq C\left(1+S(t, x+v)+S\left(t, x-v^{\prime}\right)\right. & +|\nabla S(t, x+v)|) \\
& +C \sum_{i, j=1}^{3}\left|\partial_{i j} S(t, x+v)\right| .
\end{aligned}
$$

\footnotetext{
${ }^{4}$ We have $\theta=\frac{p^{\prime}}{q^{\prime}}$ and $\epsilon=\frac{p^{\prime}}{b^{\prime}}$ therefore $\epsilon+\theta=1$ is equivalent to $\frac{1}{q^{\prime}}+\frac{1}{b^{\prime}}=\frac{1}{p^{\prime}}$. We calculate $\frac{1}{p^{\prime}}-\frac{1}{q^{\prime}}-\frac{1}{b^{\prime}}=\left(\frac{1}{q}-\frac{1}{p}\right)-1+\frac{1}{b}$. Now $-1+\frac{1}{b}=-1+\frac{5}{3}-\frac{1}{c}=\frac{2}{3}-\left(1-\theta+\frac{\theta}{q}\right)=-\frac{1}{3}+\frac{\theta}{q^{\prime}}=$ $-\frac{1}{3}+\frac{p^{\prime}}{\left(q^{\prime}\right)^{2}}$, therefore $\frac{1}{p^{\prime}}-\frac{1}{q^{\prime}}-\frac{1}{b^{\prime}}=\left(\frac{1}{q}-\frac{1}{p}\right)-\frac{1}{3}+\frac{p^{\prime}}{\left(q^{\prime}\right)^{2}}$ which is equal to zero thanks to (3.14).
} 
The obstruction in our estimates is that the proof of Theorem 1 requires $q=1$, so that the norm $\|\rho\|_{L^{q}}$ in (3.2) can be estimated a-priori, while the estimates for $f_{2}$ in the proof of Theorem 2 do not work with $q=1$ because it gives $b=3 / 2, c=1$ which is not allowed in the HLS inequality in (3.20).

\section{Global eXistence FOR SMAll DAta in the CRItiCAL NORM}

Strichartz estimates have been very successful in dealing with many classes of nonlinear Schrödinger, wave and other dispersive equations. Typically they are used to show either local existence of solutions with low regularity data or global existence under an additional smallness assumption on the initial data, see [Tao].

Proof of Theorem 3. To simplify the notation we use again the standard abbreviations for mixed spaces, for example $L_{t}^{r} L_{x}^{p} L_{v}^{q}$. In all cases the space variable $x$ runs through all of $\mathbb{R}^{3}$ and the velocity variables $v$ and $v^{\prime}$ always vary in the velocity space $V$.

Observe first that hypothesis (1.5) implies that for all $p_{1}, p_{2}, p_{3} \in[1, \infty]$ with $p_{1} \geq p_{2}, p_{3}$ we have

$$
\left\|T[S]\left(t, x, v, v^{\prime}\right)\right\|_{L_{x}^{p_{1}} L_{v}^{p_{2}} L_{v^{\prime}}^{p_{3}}} \leq C\left(|V|, p_{2}, p_{3}\right)\left[\|S(t, \cdot)\|_{L^{p_{1}}}+\|\nabla S(t, \cdot)\|_{L^{p_{1}}}\right] .
$$

Indeed, since $p_{1} \geq p_{2}, p_{3}$, we can use Minkowski's inequality to obtain

$$
\|S(t, x+v)\|_{L_{x}^{p_{1}} L_{v}^{p_{2}} L_{v^{\prime}}^{p_{3}}} \leq\|S(t, x+v)\|_{L_{v}^{p_{2}} L_{v^{\prime}}^{p_{3}} L_{x}^{p_{1}}}=C(|V|)\|S(t, \cdot)\|_{L^{p_{1}}}
$$

with similar estimates for all other terms in the right hand side of (1.5). From now on the proof will use estimate (4.1) instead of hypothesis (1.5). We present a bootstrap argument for the solution $f$ in the space $L_{t}^{3} L_{x}^{p} L_{v}^{q}$. The existence result of Theorem 3 then follows by standard methods.

As usual we have:

$$
f(t, x, v) \leq f_{1}(t, x, v)+f_{2}(t, x, v)
$$

where $f_{1}(t, x, v)$ solves

$$
\partial_{t} f_{1}+v \cdot \nabla_{x} f_{1}=0 \quad, \quad f_{1}(0, x, v)=f_{0}(x, v)
$$


and $f_{2}(t, x, v)$ solves

$$
\partial_{t} f_{2}+v \cdot \nabla_{x} f_{2}=\int_{V} T[S] f^{\prime} d v^{\prime} \quad, \quad f_{2}(0, x, v)=0 .
$$

Recall that $a \in[3 / 2,2]$. Choose $r=3$ and define $p \in[9 / 5,18 / 7]$ and $q \in[9 / 7,18 / 11]$ by $\frac{1}{p}=\frac{1}{a}-\frac{1}{9}$ and $\frac{1}{q}=\frac{1}{a}+\frac{1}{9}$. It is easy to verify that the quadruplet $(r, p, q, a)$ satisfies the conditions (2.6) required for applying the Strichartz estimates. Apply estimate (2.8) to $f_{1}(t, x, v)$ and estimate (2.10) to $f_{2}(t, x, v)$ to get:

$$
\begin{aligned}
\|f\|_{L_{t}^{3} L_{x}^{p} L_{v}^{q}} & \leq\left\|f_{1}\right\|_{L_{t}^{3} L_{x}^{p} L_{v}^{q}}+\left\|f_{2}\right\|_{L_{t}^{3} L_{x}^{p} L_{v}^{q}} \\
& \leq C\left\|f_{0}\right\|_{L_{x, v}^{a}}+C\left\|\int_{V} T[S] f^{\prime} d v^{\prime}\right\|_{L_{t}^{3 / 2} L_{x}^{q} L_{v}^{p}} .
\end{aligned}
$$

To estimate the last term in (4.3), apply first Hölder's inequality to get:

$$
\int_{V} T[S]\left(t, x, v, v^{\prime}\right) f\left(t, x, v^{\prime}\right) d v^{\prime} \leq\left\|T[S]\left(t, x, v, v^{\prime}\right)\right\|_{L_{v^{\prime}}^{q^{\prime}}}\left\|f\left(t, x, v^{\prime}\right)\right\|_{L_{v^{\prime}}^{q}} .
$$

Taking the $L_{v}^{p}$-norm of both sides we find:

$$
\left\|\int_{V} T[S] f^{\prime} d v^{\prime}\right\|_{L_{v}^{p}} \leq\left\|T[S]\left(t, x, v, v^{\prime}\right)\right\|_{L_{v}^{p} L_{v^{\prime}}^{q^{\prime}}}\left\|f\left(t, x, v^{\prime}\right)\right\|_{L_{v^{\prime}}^{q}}
$$

Taking next the $L_{x}^{q}$-norm of both sides and using Hölder's inequality with $\frac{1}{q}=\frac{1}{p}+\frac{2}{9}$ we find:

$$
\left\|\int_{V} T[S] f^{\prime} d v^{\prime}\right\|_{L_{x}^{q} L_{v}^{p}} \leq\left\|T[S]\left(t, x, v, v^{\prime}\right)\right\|_{L_{x}^{9 / 2} L_{v}^{p} L_{v^{\prime}}^{q^{\prime}}}\left\|f\left(t, x, v^{\prime}\right)\right\|_{L_{x}^{p} L_{v^{\prime}}^{q}} .
$$

It is easy to check that $\frac{9}{2} \geq p$ and $\frac{9}{2} \geq q^{\prime}$, therefore we can use (4.1) to get

$$
\begin{aligned}
\left\|T[S]\left(t, x, v, v^{\prime}\right)\right\|_{L_{x}^{9 / 2}\left(L_{v}^{p}\left(L_{v^{\prime}}^{q^{\prime}}\right)\right)} & \leq C\left(|V|, p, q^{\prime}\right)\left[\|S(t, x)\|_{L_{x}^{9 / 2}}+\|\nabla S(t, x)\|_{L_{x}^{9 / 2}}\right] \\
& =C\left(|V|, p, q^{\prime}\right)\left[\|G * \rho(t)\|_{L_{x}^{9 / 2}}+\|\nabla G * \rho(t)\|_{L_{x}^{9 / 2}}\right]
\end{aligned}
$$

where $G$ is the Bessel potential (see the proof of theorem 1).

If $\frac{9}{5}<p$ we proceed by using Young's inequality. One can show that $G \in L^{b}$ for all $b<3$ and that $\nabla G \in L^{b}$ for all $b<\frac{3}{2}$. Define $b$ by $1+\frac{2}{9}=\frac{1}{b}+\frac{1}{p}$. Then $\frac{6}{5} \leq b<\frac{3}{2}$. We get

$$
\begin{aligned}
\|G * \rho\|_{L_{x}^{9 / 2}}+\|\nabla G * \rho\|_{L_{x}^{9 / 2}} & \leq\|G\|_{L^{b}}\|\rho\|_{L_{x}^{p}}+\|\nabla G\|_{L^{b}}\|\rho\|_{L_{x}^{p}} \\
& \leq C(b)\|\rho(t, x)\|_{L_{x}^{p}} \\
& \leq C(b, q,|V|)\|f(t, x, v)\|_{L_{x}^{p} L_{v}^{q}} .
\end{aligned}
$$


If $\frac{9}{5}=p$ we use Young's inequality for the $G$-term and the HLS inequality for the $\nabla G$-term. Defining $b$ as above now gives $b=\frac{3}{2}<3$ therefore

$$
\|G * \rho\|_{L_{x}^{9 / 2}} \leq\|G\|_{L^{3 / 2}}\|\rho\|_{L_{x}^{p}} \leq C\|\rho\|_{L_{x}^{p}} \leq C(q,|V|)\|f(t, x, v)\|_{L_{x}^{p} L_{v}^{q}}
$$

One can show that $|\nabla G(x)| \leq \frac{C}{|x|^{2}}$ for all $x$. Therefore, by HLS, $\|\nabla G * \rho\|_{L_{x}^{9 / 2}} \leq\left\|\frac{C}{|x|^{2}} * \rho\right\|_{L_{x}^{9 / 2}} \leq C\|\rho\|_{L_{x}^{9 / 5}}=C\|\rho\|_{L_{x}^{p}} \leq C(q,|V|)\|f(t, x, v)\|_{L_{x}^{p} L_{v}^{q}}$.

The above argument shows that

$$
\left\|T[S]\left(t, x, v, v^{\prime}\right)\right\|_{L_{x}^{9 / 2} L_{v}^{p} L_{v^{\prime}}^{q^{\prime}}} \leq C(a,|V|)\|f(t, x, v)\|_{L_{x}^{p} L_{v}^{q}} .
$$

Using (4.6) into (4.4) we get:

$$
\left\|\int_{V} T[S] f^{\prime} d v^{\prime}\right\|_{L_{x}^{q} L_{v}^{p}} \leq C(a,|V|)\|f(t, x, v)\|_{L_{x}^{p} L_{v}^{q}}^{2} .
$$

Taking the $L_{t}^{3 / 2}$-norm of both sides we obtain:

$$
\left\|\int_{V} T[S] f^{\prime} d v^{\prime}\right\|_{L_{t}^{3 / 2} L_{x}^{q} L_{v}^{p}} \leq\|\| f(t, x, v)\left\|_{L_{x}^{p}\left(L_{v}^{q}\right)}^{2}\right\|_{L_{t}^{3 / 2}}=\|f(t, x, v)\|_{L_{t}^{3} L_{x}^{p} L_{v}^{q}}^{2}
$$

Using this in (4.3) we find

$$
\|f\|_{L_{t}^{3} L_{x}^{p} L_{v}^{q}} \leq C\left\|f_{0}\right\|_{L^{a}\left(\mathbb{R}^{6}\right)}+C\|f(t, x, v)\|_{L_{t}^{3} L_{x}^{p} L_{v}^{q}}^{2} .
$$

This completes the a-priori estimates which enable to bootstrap for small initial data. See remark 4.2 .

Remark 4.1. The proof of Theorem 3 works in $d=4$ dimensions too. One may choose for instance $(q, p, r, a)=(3,12 / 5,12 / 7,2)$. Notice that $a=\frac{d}{2}$. It also works in dimension $d=2$, however, in this case a better result (global existence without a smallness assumption) is available in [HKS1.

Using the same method we can prove local existence for large data.

Remark 4.2. We have proved a priori estimates for the IVP (1.1). We can prove the existence of weak solutions using regularization and compactness. In particular the compactness can be gained using averaging lemmas (see [P2] for instance) provided we get some a priori estimate on the $L^{p} L^{q}$-norm of $f$ with $q>1$. This has been obtained in the proofs of theorems 2 and 3 , whereas in theorem 1 an additional bootstrap step is needed. Of course continuity of $T[S]$ in spaces $L_{l o c}^{p}$ is needed for passing to the limit in all cases. 


\section{Conclusions AND PERspectives}

In this paper we have considered a number of classes of turning kernels in the kinetic model of chemotaxis. We have proved global existence for arbitrarily large data using dispersion estimates for several of them, and, using Strichartz estimates, we have obtained global existence for small solutions in the most difficult case of a turning kernel that involves $|\nabla S(t, x+v)|+\left|\nabla S\left(t, x-v^{\prime}\right)\right|$ (Theorem 3). The de-localization induced by $v$ or $v^{\prime}$ in these formula is fundamental both for mathematical theory and biophysical interpretation. However, several questions remain that show that the present theory still needs to be improved. We would like to mention a few of them.

At first, obviously is the case of large initial data in Theorem 3 which remains open. Notice that the time integrability in the Strichartz estimates implies some decay to zero at infinity which is only possible for small initial data, as we know from the Keller-Segel system [BDP, CPZ].

A second question is to include some of these examples in a more general assumption such as

$$
\left\|T[S]\left(t, x, v^{\prime}, v\right)\right\|_{L_{l o c}^{\infty}} \leq C\|S\|_{L_{l o c}^{\infty}}
$$

Because this does not include directly de-localization, the methods used here do not apply for global existence.

More related to biophysical interpretation there is a third question: unlike in the Keller-Segel model - where having turning kernels of the form $S\left(t, x+v^{\prime}\right)$ and $\nabla S\left(t, x+v^{\prime}\right)$ gives a repellent drift [CMPS] and then there is no blow-up, and the existence theory is much simpler - in the arguments carried out in the proof of the results we do not see why it should be better to have turning kernels of the latter form.

Acknowledment. The authors would like to acknowledge support from European Networks MRNTN-CT-2004-503661, HPRN-CT-2002-00282, and HPRN-CT-200100273 - HARP. 


\section{REFERENCES}

[Alt] W. Alt, Biased random walk models for chemotaxis and related diffusion approximations, J. Math. Biol. 9, 147-177 (1980).

[BDP] A. Blanchet, J. Dolbeault and B. Perthame, Two-dimensional Keller-Segel model: optimal critical mass and qualitative properties of the solutions, Electron. J. Diff. Eqns. 2006(44), 1-33 (2006).

[CP] F. Castella and B. Perthame, Estimations de Strichartz pour les équations de transport cinétique, C. R. Acad. Sci. Paris Sér. I 322(6), 535-540 (1996).

[CPZ] L. Corrias, B. Perthame and H. Zaag, Global solutions of some chemotaxis and angiogenesis systems in high space dimensions, Milan J. Math. 72, 1-28 (2004).

[CMPS] F.A.C.C. Chalub, P.A. Markowich, B. Perthame and C. Schmeiser, Kinetic models for chemotaxis and their drift-diffusion limits, Monatsh. Math. 142, 123-141 (2004).

[CR] F.A.C.C. Chalub and J.F. Rodrigues, A class of kinetic models for chemotaxis with threshold to prevent overcrowding, Portugaliae Mathematica 63(2), 1-24 (2006).

[DS] Y. Dolak and C. Schmeiser, A kinetic theory approach for resolving the chemotactic wave paradox, Math. Mod. Comput. Biol. Med., 171-177, Milan Res. Cent. Ind. Appl. Math. MIRIAM Proj., 1, Esculapio, Bologna (2003).

[EH] R. Erban and H.J Hwang, Global existence results for complex hyperbolic models of bacterial chemotaxis, preprint arXiv:math.AP/0602139 (2006).

[FLP] F. Filbet, P. Laurençot and B. Perthame, Derivation of hyperbolic models for chemosensitive movement, J. Math. Biol. 50(2), 189-207 (2005).

[GL] R.T. Glassey, The Cauchy problem in kinetic theory, SIAM publications, Philadelphia 1996.

[HO] T. Hillen and H.G. Othmer, The diffusion limit of transport equations derived from velocity-jump processes, SIAM J. Appl. Math. 61(3), 751-775 (2000).

[HPS] T. Hillen, K.J. Painter and C. Schmeiser, Global existence for chemotaxis with finite sampling radius, preprint (2005).

[H] D. Horstmann, From 1970 until present : the Keller-Segel model in chemotaxis and its consequences I., Jahresber. Deutsch. Math.-Verein. 105, 103-165 (2003).

[HKS1] H.J. Hwang, K. Kang and A. Stevens, Global solutions of nonlinear transport equations for chemosensitive movement, SIAM J. Math. Anal. 36(4), 1177-1199 (2005).

[HKS3] H.J. Hwang, K. Kang and A. Stevens, Drift-diffusion limits of kinetic models for chemotaxis: a generalization, Discrete Contin. Dyn. Syst. Ser. B 5(2), 319-334 (2005).

[HKS2] H.J. Hwang, K. Kang and A. Stevens, Global existence of classical solutions for a hyperbolic chemotaxis model and its parabolic limit, Indiana Univ. Math. J. 55(1), 289-316 (2006).

[KS1] E.F. Keller and L.A. Segel, Initiation of slide mold aggregation viewd as an instability, J. Theor. Biol. 26, 399-415 (1970). 
[KS2] E.F. Keller and L.A. Segel, Model for chemotaxis, J. Theor. Biol. 30, 225-234 (1971).

[KS3] E.F. Keller and L.A. Segel, Traveling bands of chemotactic bacteria: a theoretical analysis, J. Theor. Biol. 30, 235-248 (1971).

[ODA] H.G. Othmer, S.R. Dunbar and W. Alt, Models of dispersal in biological systems, J. Math. Biol. 26(3), 263-298 (1988).

[P1] B. Perthame, PDE models for chemotactic movements: parabolic, hyperbolic and kinetic, Appl. Math. 49(6), 539-564 (2004).

[P2] B. Perthame, Mathematical tools for kinetic equations, Bull. Amer. Math. Soc. (N.S.) 41(2), 205-244 (2004).

[S] E.M. Stein, Singular integrals and differentiability properties of functions, Princeton Mathematical Series, No. 30 Princeton University Press, Princeton, N.J. 1970.

[Tao] T. Tao, Nonlinear Dispersive Equations: Local and Global Analysis, AMS, 2006.

University of Edinburgh, School of Mathematics, JCMB, King's Buildings, Edinburgh EH9 3JZ, UK

E-mail address: N.Bournaveas@ed.ac.uk

École Normale Supérieure, Département de mathématiques et applications, CNRS UMR8553, 45 Rue D'Ulm, F 75230, Paris, Cedex 05, France

E-mail address: Vincent.Calvez@ens.fr

University of Nottingham, School of Mathematical Sciences, Division of TheoretiCal Mechanics, University of Nottingham, University Park, Nottingham NG7 2RD, UK

E-mail address: susana.gutierrez@nottingham.ac.uk

École Normale Supérieure, Département de mathématiques et applications, CNRS UMR8553, 45 Rue D'Ulm, F 75230, Paris, cedex 05, France

E-mail address: Benoit.Perthame@ens.fr 